\title{
Immune Checkpoint Inhibition in Classical Hodgkin Lymphoma: From Early Achievements towards New Perspectives
}

\author{
Diego De Goycoechea, ${ }^{1}$ Gregoire Stalder, ${ }^{1}$ Filipe Martins ${ }^{(1 D},{ }^{1,2}$ and Michel A. Duchosal ${ }^{1}$ \\ ${ }^{1}$ Service and Central Laboratory of Hematology, Centre Hospitalier Universitaire Vaudois and University of Lausanne, \\ rue du Bugnon 46, CH-1011 Lausanne, Switzerland \\ ${ }^{2}$ School of Life Sciences, Ecole Polytechnique Fédérale de Lausanne (EPFL), 1015 Lausanne, Switzerland
}

Correspondence should be addressed to Filipe Martins; filipe.martins@epfl.ch

Received 18 October 2018; Revised 18 March 2019; Accepted 17 April 2019; Published 7 May 2019

Guest Editor: Shalini Gupta

\begin{abstract}
Copyright ( 2019 Diego De Goycoechea et al. This is an open access article distributed under the Creative Commons Attribution License, which permits unrestricted use, distribution, and reproduction in any medium, provided the original work is properly cited.

Immune checkpoint inhibition (ICI) became one of the major breakthroughs in cancer treatment over the past decade and entered into therapy within standard oncohematology practice. ICI has demonstrated impressive response rates as salvage therapy in relapsed/refractory $(\mathrm{R} / \mathrm{R})$ classical Hodgkin lymphoma $(\mathrm{cHL})$ and is now being tested as an adjunction to chemotherapy in the frontline settings. CHL exquisite sensitivity to PD-1/PD-L1 axis inhibition relies on a particular biological background. By contrast, non-Hodgkin lymphomas (NHL) have demonstrated heterogeneous response rates using ICI. These observations highlight discrepancies between various types of lymphomas in terms of genetic alterations, immune microenvironment interactions, and disease phenotype. This review aims to focus on $\mathrm{cHL}$ immune escape mechanisms, focusing on cHL biological sensitivity to PD-1 blockade. We will summarize the available data issued from clinical trials on ICI in cHL and its safety profile. Going beyond the current use of monoclonal antibodies (mAb) targeting immune checkpoints in clinical practice, we will offer an overview of new combinatory therapeutic perspectives where $\mathrm{cHL}$ immunotherapy may be considered.
\end{abstract}

\section{Introduction}

Accounting for a tenth of lymphoma cases, classical Hodgkin lymphoma (cHL) is characterized by peculiar histologic and immunologic features [1]. A striking inflammatory infiltrate surrounding rare multinucleated giant cells were originally reported by Dorothy Reed more than one hundred years ago. This observation already highlighted the intriguing immune repercussion of $\mathrm{cHL}$ where authors noticed tuberculin anergy in affected patients [2]. CHLassociated cellular immunosuppression, which translates into an increased infectious risk that may precede disease by several years, was also further supported by the necessity of delivering irradiated blood products to avoid the risk of transfusion-associated graft-versus host disease (GVHD) in these patients [3]. Although considered a curable disease in almost $80 \%$ of cases, relapse cases of cHL are still challenging. Rescue and intensive chemotherapies followed by autologous hematopoietic stem cell transplantation
(auto-HSCT) can put into remission about half of the patients [4].

The subset of patients necessitating further treatment in the cases of second relapse or refractory disease is considered for allogeneic HSCT (allo-HSCT). In this situation, a survival plateau has been difficult to reach at least until recently [5].

The impressive results of nivolumab (a fully human IgG4 monoclonal antibody against PD-1) in $\mathrm{R} / \mathrm{R}$ cHL led to its FDA approval in $2016[6,7]$. Demonstrating an objective response rate (ORR) of $66.3 \%$ in the Checkmate 205 trial, nivolumab's unprecedented performance made it a robust alternative bridge therapy to allo-HSCT [8]. CHL exquisite sensitivity to PD-1 blockade relies on lymphoma cell genetic alterations and particular tumor microenvironment (TME) inflammatory phenotype. In an attempt to optimize the firstline treatment of cHL, PD-1 blockade is now being tested as an adjunct to doxorubicin, vinblastine, and dacarbazine ("AVD" regimen) in Europe and USA in both early and advanced stages (NCT03004833 and NCT03033914 trials), respectively. 
Preliminary results have shown high response rates with an acceptable safety profile in the frontline setting with patients achieving complete responses (CR) in $67-80 \%$ of cases $[9,10]$. High expectations regarding PD-1 blockade in $\mathrm{cHL}$ reside in its potential to decrease treatment-related toxicity of current intensive chemotherapy regimen, such as bleomycin-related pulmonary toxicity, and to challenge the place of adjuvant radiotherapy in affected young populations [11]. Avoiding bleomycin may reduce the rate of fatal pulmonary toxicities, which has been reported in $4-5 \%$ of $\mathrm{cHL}$ patients in a systematic review [11]. Anti-PD-1 mAb are also being studied as an upfront treatment in patients unsuitable for standard therapy (NCT03331731).

Results of PD-1 blockade efficacy in R/R NHL are more variable: it is effective to treat primary mediastinal Bcell lymphoma (PMBCL) [12], Grey-Zone lymphoma [13], CNS primary diffuse large B-cell lymphoma (CNS-DLBCL), and primary testicular lymphoma (PTL) [14], where PD1 inhibition significantly affects response rates. Heterogeneous immune escape pathways' expression and variable immunosuppressive properties of NHL explain in part these disparities [15].

Focusing on $\mathrm{cHL}$ as a paradigm for its high sensitivity to ICI, this review brings insights into the biological background behind its effectiveness. It also reflects on ICI current place in patient care and provides an overview of the strategies being foreseen to boost its effects in the future.

\section{2. cHL Microenvironment and Immune Escape Mechanisms}

CHL is a malignancy issued from giant, often multinucleated cells, called Reed-Sternberg (HRS). These cells constitute less than $5 \%$ of the tumor bulk, and they grow and survive with the help of interactions with and within a heterogeneous background of inflammatory cells. Germinal center B-cells are considered to be the origin of HRS cells [16].

In the era of polychemotherapy and radiation therapy, the various subtypes of $\mathrm{cHL}$, namely, nodular sclerosis, lymphocyte-rich, lymphocyte-depleted, and mixed cellularity subtypes, demonstrating the heterogeneity of their underlying biology, do not translate into direct consequences for patient care [17]. The latter is mainly driven by disease stage and other risk features [18, 19]. However, the underlying biology of cHL offers now new prognostic markers and may regain the interest of clinicians in this new era of immunotherapy. Collectively, these histological subtypes share a variable number of infiltrating lymphocytes, generally forming the main component of the tumor cell environment, monocyte-macrophages, eosinophils, neutrophils, plasma cells, and mesenchymal stromal cells (MSC), such as endothelial cells and fibroblasts associated with a variable degree of tissue extracellular matrix. These infiltrative components are necessary to promote HRS growth. This has been demonstrated by HRS absence of growth in ex vivo experiments and indirectly by substantial difficulties to establish cell lines where interstitial cells are lacking [20]. Reciprocally, HRS cells shape their microenvironment to benefit in return from growth and survival signals provided by surrounding inflammatory cells. Directly and indirectly, HRS attract surrounding cells via chemokine secretion. This is valid for neutrophils (IL-8), eosinophils (CCL5, eotaxin), macrophages (CCL5), mast cells (CCL5), T regulatory lymphocytes (CCL5, CCL17, CCL20, CCL22), and type 2 helper T-cells (CCL17, CCL22). These environmental cells provide not only survival/support signals for HRS, but also, for part of them, immune escape signals [21-24].

Main HRS survival signals lead to NF-kB pathway hyperactivation [25]. They originate from CD40, CD30, TACI, and BCMA receptors engagement by their respective ligands located on the surface of surrounding CD4+ T-cells, eosinophils and mast cells (i.e. CD40L and CD30L), and/or secreted molecules from myeloid-derived cells and neutrophils for BAFF and APRIL, respectively [26, 27].

To counteract immune tumor rejection, HRS shape the TME to induce immune tolerance (Figure 1). HRS cells are coated with a large variety of immune checkpoint ligands and transmembrane receptors mediating exhaustion of infiltrating cytotoxic and type 1 helper T-cell subsets. Cytotoxic T-lymphocyte antigen 4 (CTLA-4), membrane-bound TGF$\beta$, and PD-L1 (B7-H1) expression are some of the main Treg contact mediators acting in this exhaustion process. Among these, $\mathrm{PD}-1 / \mathrm{PD}-\mathrm{L} 1$ axis constitutes one of the major escape mechanisms in $\mathrm{cHL}$, as demonstrated by its dense coating on HRS cells [28], which is linked to a high prevalence of 9p24.1 chromosomic amplification, a loci bearing PD-L1/2 genes [29]. A similar amplification is also frequent in some groups of NHL such as PMBCL, CNS-DLBCL, and PTL cases, thus explaining in part their higher relative sensitivity towards ICI when compared to other B-cell NHL. Chromosomal 9p24.1 copy number gains and gene amplifications also affect nearby JAK2 locus, which further intensifies PD-L1 overexpression through JAK/STAT signal pathway activation. Latent membrane protein 1 (LMP1) encoded by the inserted EBV genome mimics CD40 signaling and therefore amplifies PD-L1/L2 overexpression, through AP-1 and JAK/STAT3mediated epigenetic control. This observation sustains a reciprocal positive biological feedback between avoidance of viral clearance and immune escape of HRS cells [30-32]. PD1-PD-L1/2 ligation triggers $\mathrm{T}$-cell phosphatase activation and consequent dephosphorylation cascade of several proteins implicated in T-cell receptor (TCR), and PI3K-AKT-to-NF$\mathrm{kB}$ signaling pathways. Tyrosine phosphatases are actually recognized as important immune checkpoint modulators, and active research on their potential inhibition to boost adoptive T-cell therapy is ongoing [33]. Consequently, IL2 and IFN $\gamma$ secretion is also repressed, thus inhibiting Tcell cytolytic activity and cell proliferation. Stanford's pathology department recently published their evaluation of PDL1/-L2 expression on 702 immunostained B-/T-lymphoma samples [28]. This study confirmed the high prevalence of PD-L1 positivity in cHL samples (over $80 \%$ in $\mathrm{cHL}$ and $75 \%$ in nodular lymphocyte-predominant HL (NLPHL), resp.). In this series, all except one PD-L1 positive cHL sample (40 over 41) were also Epstein-Barr (EBV) positive by EBER in situ hybridization, in opposition to three over the nine PD-L1negative cHL samples. 


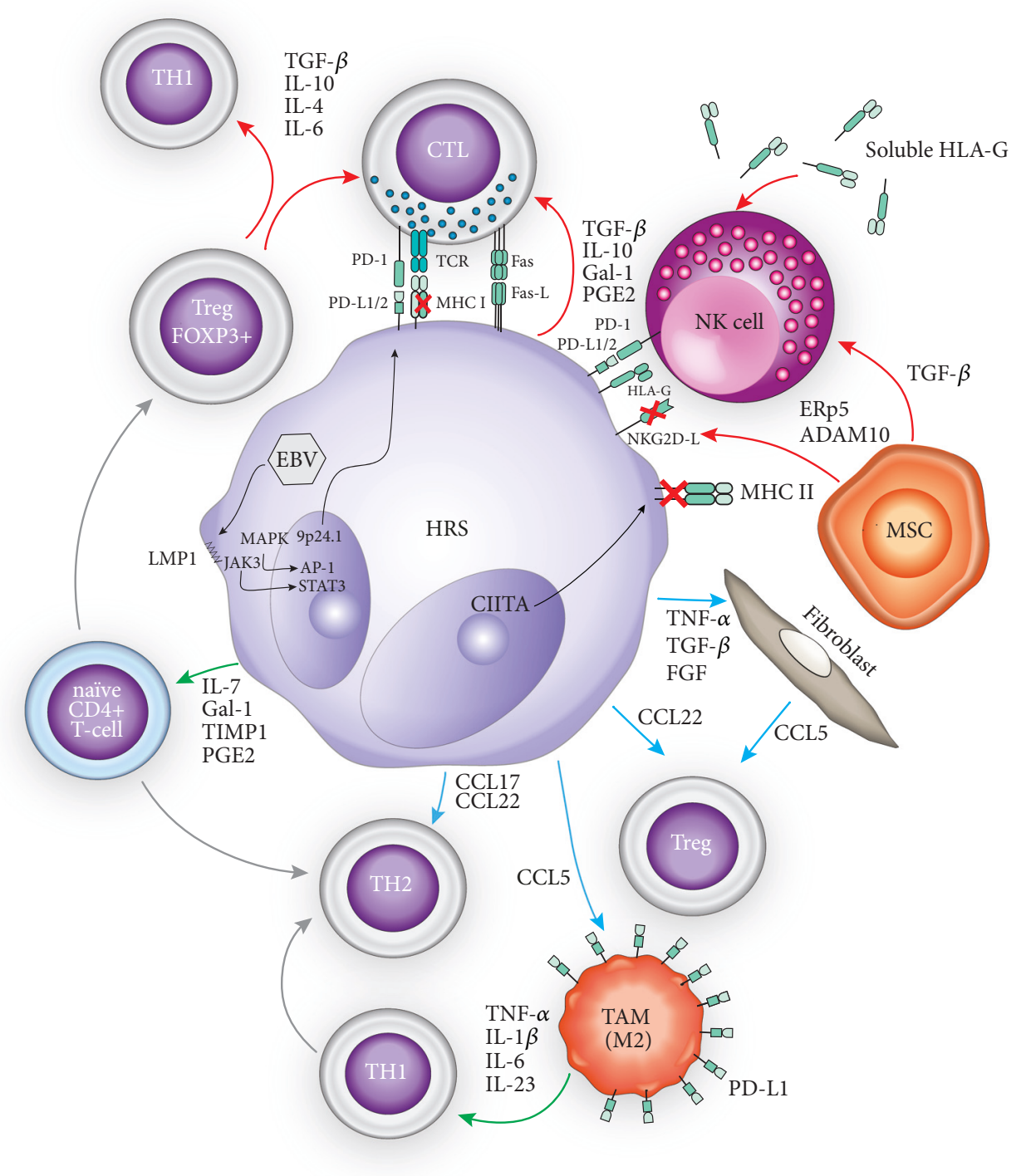

FIGURE 1: Immune-escape mechanisms of cHL TME. Chemokine secretion by HRS plays a central role in the TME immunosuppressive state of cHL. They allow differentiation of infiltrating naïve CD4+ T-cells into regulatory FOXP3+ and Th2 T-cells and provide CTL inhibitory signals through TGF- 3 , IL-10, galectin-1 (Gal-1), tissue inhibitor of metalloproteinase 1 (TIMP1), and prostaglandin E2 (PGE2). HRS also attract Tregs/Th2 T-cells from the systemic circulation, through the secretion of CCL22 and CCL17, respectively, while at the same time promoting their expansion through the secretion of Gal-1, TIMP1, and PGE2. Fibroblasts also contribute to Treg chemoattraction through CCL5 secretion. In the same line, TAM promote the differentiation of Th1 cells towards the Th2 phenotype. Nevertheless, recent evidence challenges this concept of predominant Th2 polarized TME and evidenced an increase in activated Th1 T-cells in TME of cHL patients. EBV latent infection also plays a key role, through the production of LMP1 which activates the MAPK and JAK/STAT3 pathways leading to transcriptional activation of the 9p24.1 locus with consequent PD-L1/2 overexpression [5]. Even in EBV negative cHL, PD-L1 (and, to a lesser degree, PD-L2) expression at the surface of HRS cells is still high. PD-1-PD-L1 interaction triggers the inhibition of CTL function. TAM display a high surface PD-L1 expression, thus promoting PD-1-PD-L1 axis immune escape. EBV- cHL displays also a decrease in MHC class I expression, in comparison to their EBV positive counterparts mainly through B2-microglobulin subunit downregulation. MHC class II expression can also be impaired through epigenetic silencing, in the subset of mutated/translocated CIITA cHL. In this context, it is presumed that the PD-1/PD-L1/2 axis mediates immune escape, in first instance, by dampening NK cell activity. NK function is further downregulated by TGF- $\beta$ secreted by HRS and mesenchymal MSC. MSC also edit the surface expression of NKG2D-L through the enzymatic activity of secreted ERp5 and ADAM10. HRS cells also express Fas ligand at their surface, thus promoting apoptosis of interacting Th1 and cytotoxic T-cells [88]. Red arrows: "inhibition signal"; green arrows: "differentiation signal"; blue arrows: "chemoattraction" signal. 
TABLE 1: Summary of immune escape mechanisms in cHL and alternative therapeutic strategies in development.

\begin{tabular}{|c|c|}
\hline Immune escape mechanisms in $\mathrm{cHL}$ & $\begin{array}{c}\text { Therapeutic agents with immunomodulatory properties tested in } \\
\text { recruiting/active clinical trials in } \mathrm{R} / \mathrm{R} \mathrm{cHL}\end{array}$ \\
\hline Downregulation of MHC class I and II expression & $\begin{array}{c}\text { Epigenetic modifiers in combination with immune checkpoint inhibitors } * \text { : } \\
\text { Decitabine + anti-PD-1 mAbs (NCT03250962) }\end{array}$ \\
\hline Surface PD-L1/2 overexpression & $\begin{array}{c}\text { JAK/STAT inhibitors in combination with immune checkpoint inhibitors: } \\
\text { Ruxolitinib + anti-PD-1 mAbs (NCT03681561) } \\
\text { Combinatorial immune checkpoint blockade: } \\
\text { Ipilimumab + Nivolumab (NCT02408861, NCT02304458) } \\
\text { Anti-LAG3 mAb (MK-4280) + anti-PD-1 mAb (NCT03598608) } \\
\text { Brentuximab + Nivolumab +/- Ipilimumab (NCT01896999) }\end{array}$ \\
\hline $\begin{array}{l}\text { CTL anergy through PD-1-PD-L1/2 interaction (HRS / } \\
\text { TAM). }\end{array}$ & $\begin{array}{c}\text { Adoptive cell therapy: } \\
\text { Chimeric Antigen Receptor (CAR) CD30-targeting T-cells (NCT01316146, } \\
\text { NCT01192464, NCT02690545, NCT02917083, NCT02259556, NCT03602157, } \\
\text { NCT03049449) } \\
\text { Bi-specific chimeric antibody constructs: } \\
\text { INBRX-105 (PD-L1-CD137) provides a combination of PD-L1 blockade with } \\
\text { concomitant T-cell co-stimulation through CD137 (4-1BB) agonism } \\
\text { (NCT03809624) }\end{array}$ \\
\hline $\begin{array}{l}\text { NK cell inhibition mediated by TGF-ß and NKG2D-L } \\
\text { interaction (HRS / MSC). }\end{array}$ & $\begin{array}{c}\text { Bi-specific chimeric antibody constructs: } \\
\text { AFM13 (CD30-CD16A) recruits NK cells via binding to CD16A as immune } \\
\text { effector cells (NCT02321592) } \\
\text { AFM13 + anti-PD-1 mAbs (NCT02665650) }\end{array}$ \\
\hline
\end{tabular}

CTL inhibition through TGF-ß, IL-10, Gal-1, TIMP1

and PGE2.

Stimulation of CD4 T-cells differentiation towards Treg and Th2 phenotype through TGF- 3 , IL-10, Gal-1, TIMP1 and PGE2.

Chemo attraction of Treg and Th2 through CCL5

(fibroblasts), CCL17 and CC22 (HRS).

Th1 and CTL enhanced apoptosis through Fas ligand surface expression (HRS).

Immunomodulatory agents:

Lenalidomide + anti-PD-1 mAbs (NCT02875067, NCT03015896, NCT01953692) Ibrutinib + anti-PD-1 mAbs (NCT02940301). Induction of immunogenic cell death (ICD) of tumor cells with chemotherapy in combination with immune checkpoint inhibitors:

Bendamustine + anti-PD-1 mAbs (NCT03343652)

Bendamustine + Gemcitabine + anti-PD-1 mAbs (NCT03739619)

* It should be noted that the trials involving inhibitors of deacetylase (HDACi) in cHL revealed a limited efficacy with significant hematological and electrolytic toxicities, rendering their future development difficult in the absence of predictive biomarkers $[89,90]$.

CTLA-4 is another immune checkpoint located on the surface of T-cells which dampers the priming (early) phase of immune response. This function makes CTLA-4 a natural synergistic partner of PD-1/PD-L1 inhibition. Indeed, in addition to impeding CD28 costimulatory signaling in barring T-cells, by competing for its ligands (CD80/CD86), CTLA-4 interaction impacts also the NF-kB pathway leading to IL-2 production impairment $[34,35]$. Its constitutive presence on Tregs also offers the possibility to target directly a main actor of TME immunosuppression [36]. Recent success in the treatment of melanoma, clear-cell renal cancer, and non-small cell lung cancer brings high expectations regarding ongoing trials combining both PD-1-PD-L1 and CTLA-4 inhibition in relapsing cHL (Table 1) [37-39].

Immunochemistry studies showed a high prevalence (>90\%) of additional immune checkpoint regulators such as lymphocyte activation gene-3 (LAG3), T cell immunoglobulin, and mucin domain-containing protein-3 (TIM3) expressions. Expressions were found mainly on T-cells composing the TME of CHL. This was in opposition to PD-L1 displaying almost universal positivity on HRS cells. TIM3 was present in only one-third of samples included in a study assessing samples from 25 cases of cHL, while HRS were weakly LAG3-positive in a single case [40]. The implications of these findings are still unclear, even though they highlight a presumed significant role of these additional immune checkpoints within the TME component. LAG3 (CD223) is a cell surface receptor expressed by Tregs, activated B/T-cells, and antigen-presenting dendritic cells, which binds with high affinity to the major histocompatibility complex (MHC) class II. Functional consequences of its ligand engagement are cell context-dependent, promoting Treg function enhancement on the one hand, and suppressing effector T-cell function on the other hand $[41,42]$. However, LAG3 signaling and intervening mechanisms of CD4/CD8 T-cell downmodulation are still poorly characterized [43]. TIM3 is an inhibitor receptor implicated in the exhaustion of cytotoxic and Th1 tumor infiltrating T-cells, although as for its former counterpart the underpinning mechanisms are still to be fully characterized $[44,45]$. One of its ligands, Galectin-9, is a mediator Th1 cell death [46]. Blockade of these regulatory components is under active clinical research with several ongoing trials in solid and hematologic neoplasms, since they displayed synergism with PD-1 blockade in a preclinical setting (Table 1). 
HRS also occults surface MHC class I and II in about 2/3 of cases, playing so, on both of the two-signal dependency of T-cell activation [47]. B2-microglobulin transcription repression constitutes the main mechanism of MHC class I downregulation and seems inversely correlated with EBV status. MHC class II is also downregulated at a transcriptional level in the subset of Class II Transactivator Type I (CIITA) mutated and/or translocated cHL. MHC class II expression negativity is found in $15 \%$ to $40 \%$ of cases [48, 49]. Altogether, one-third of cHL display no expression of both MHC. These observations led to the assumption that PD-1 blockade efficiency in cHL is not primarily related to reinforce CTL immune rejection. Instead, a more pronounced effect on reversing Natural Killer cells (NK) inactivation is presumed, by impairing the interaction of PD-1 with PD-L1 located on the surface of HRS and tumor-associated macrophages (TAM). The latter are main providers of surface PD-L1 in the cHL TME because of its high density of expression on their surface [50].

NK cells are important mediators of antitumor surveillance. However, HRS cells are resistant to Fas receptormediated death and even in the absence of most MHC class I molecules are able to avoid NK cell activation. This is mediated by the expression of surface $B 2$-microglobulin-free HLA-G subunits [51]. HLA-G is mainly expressed in the placenta and plays a crucial role in its immunotolerance. A soluble form of the latter is also secreted by HRS cells and impairs NK cell extravasation and tissue migration. Finally, HLA-G can induce Treg differentiation. HRS cells display low levels of surface NKG2D ligand through secretion of proteolytic enzymes such as ERp5 (a disintegrin) and ADAM10 (a metalloproteinase domain-containing protein 10), also produced by MSC [52]. A soluble form of the NKG2D ligand is also presumably responsible for the internalization and subsequent downregulation of its receptor on circulating NK cells, thus inducing a systemic cellular dysfunction $[53,54]$. TGF- $\beta$ secreted by MSCs further reinforces the downregulation of NKG2D receptor on the surface of NK and cytotoxic T-cells [55]. Several of the beforementioned characteristics of cHL immune escape, as, for example, a high number of infiltrating TAMs and a lack of MHC class I expression, negatively impact disease outcome $[47,56]$. A summary of these immune escape mechanisms, together with treatment strategies under clinical investigation to overcome them, is provided in Table 1.

\section{Immune Checkpoint Inhibition in cHL}

3.1. Clinical Outcomes. The preclinical observation of PD-L1 overexpression in cHL led to the evaluation of ICI administration on disease evolution. To date, seven prospective clinical studies with ICI in cHL have been published (Table 2). In the first phase 1 study published in 2015 (Checkmate 039), 23 patients with R/R cHL were treated with nivolumab $3 \mathrm{mg}$ per kilogram of body weight every 2 weeks until complete response, tumor progression, or excessive toxicity [7]. Eighteen patients had relapsed after auto-HSCT and/or received brentuximab vedotin (BV) (an antibody-drug conjugate directed against CD30 and linked to microtubule-disrupting agent monomethyl auristatin E, MMAE) before relapse. High-grade adverse events (G3-4) occurred in 12 patients. Four patients had a complete response (CR) and 16 patients had a partial response (PR). The progression-free survival (PFS) at 24 weeks was $86 \%$. Following this study, the same group of investigators performed a phase 2 study (Checkmate 205 ) including 243 patients. The latter was composed of three cohorts divided according to their treatment history: patients who did not receive $B V$ (cohort $A, n=63$ ), patients treated with BV after auto-HSCT (cohort $B, n=80$ ), and patients who were treated with BV before and/or after auto-HCT (cohort $\mathrm{C}, \mathrm{n}=100$ ). The initial results from cohort $\mathrm{B}$ were published in the Lancet Oncology in 2016 [8]. Seven patients had a CR and 46 patients had a PR, with a PFS of $76.9 \%$ at 6 months. The results of the extended follow-up of the three cohorts were published in 2018 [57]. The overall response rate (ORR) was 69\% (95\% CI, 63\% to 75\%). Forty patients had a CR and the median PFS was 14.7 months. Response rates were similar across the three cohorts. The updated results of Checkmate 205 were presented in December 2018. Actualized ORR was $71 \%$ with $21 \%$ of patients achieving CR (Cohort A $32 \%$, Cohort B 14\%, Cohort C 20\%) [58].

It should be emphasized that the Checkmate 205 study protocol was amended in July 2014 to allow patients to continue treatment beyond investigator-assessed progression if protocol-predefined criteria were met, including stable performance status and perceived clinical benefit. Patients treated beyond initial progression (TBP) were required to discontinue in the event of further progression $(>10 \%$ further increase in tumor burden) [8]. Cohen reported on the 80 patients TBP over the 130 patients with progressive disease in the Checkmate 205 study. Amongst 67 evaluable patients TBP, 37 experienced stable or reduced target tumor burdens, despite the appearance of new lesions [59].

Finally, a small phase 2 study on nivolumab in relapsed cHL after treatment with BV on 17 patients with or without previous auto-HSCT was performed in Japan [60]. CR and PR occurred in four and nine patients, respectively, with a PFS of $60 \%$ at 6 months.

Another anti-PD-1 mAb, pembrolizumab, was studied in a phase 1 trial published in 2016 (Keynote 013) [61]. Thirty-one patients, all previously treated with BV with 22 of them having also received an auto-HSCT before relapse, were treated with pembrolizumab $10 \mathrm{mg}$ per kilogram of body weight every 2 weeks. Five patients had a CR and 15 a PR, with a PFS of $69 \%$ and $46 \%$ at 24 and 52 weeks, respectively. Thereafter, a phase 2 study was conducted (Keynote 087), but with a dose of pembrolizumab of $200 \mathrm{mg}$ once every 3 weeks, based on its pharmacokinetic properties [62]. Patients were divided in three cohorts: those relapsing after auto-HSCT and subsequent BV (Cohort $1, n=69$ ), those who were ineligible for auto-HSCT because of refractoriness to salvage chemotherapy and BV (Cohort 2, n=81), and those relapsing after auto-HSCT but without subsequent BV (although $41.7 \%$ received BV before transplantation) (Cohort $3, \mathrm{n}=60$ ). Among the 210 included patients, the ORR was $69 \%$ with $22.4 \%$ of CR. PFS at 6 months was $72.4 \%$. There were no significant differences between the three cohorts. The updated results presented at ASH 2018 showed an ORR of 


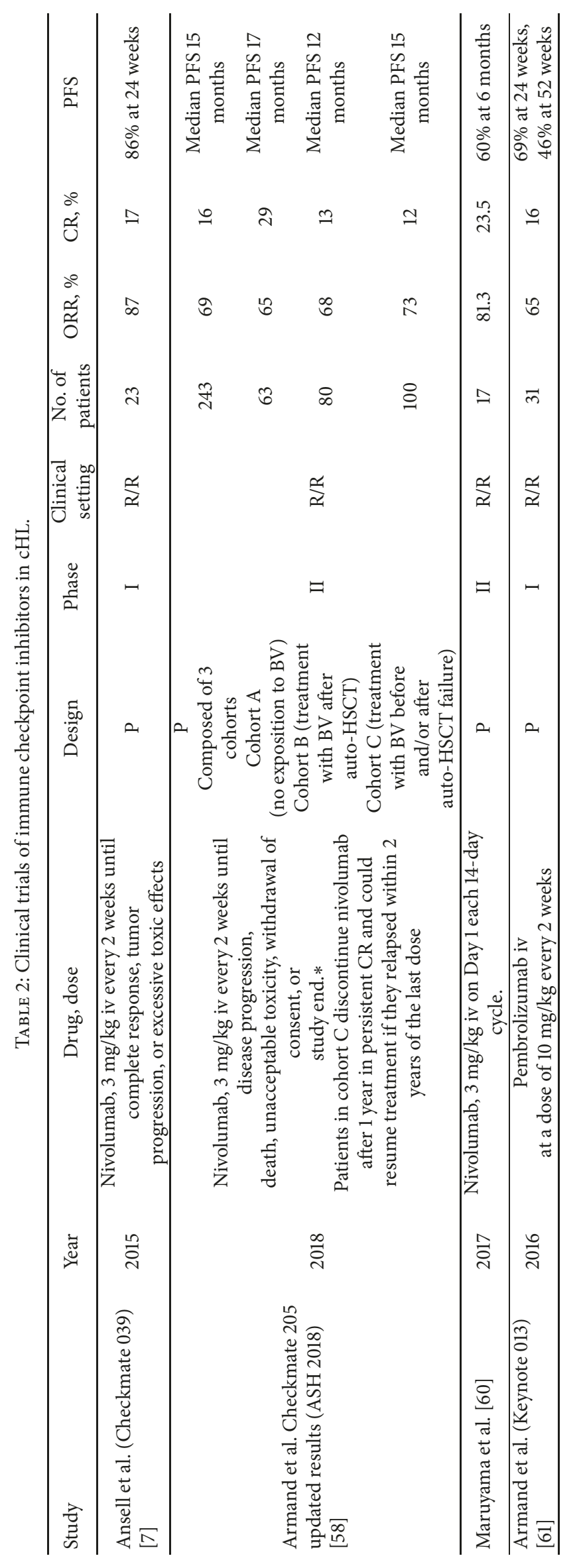




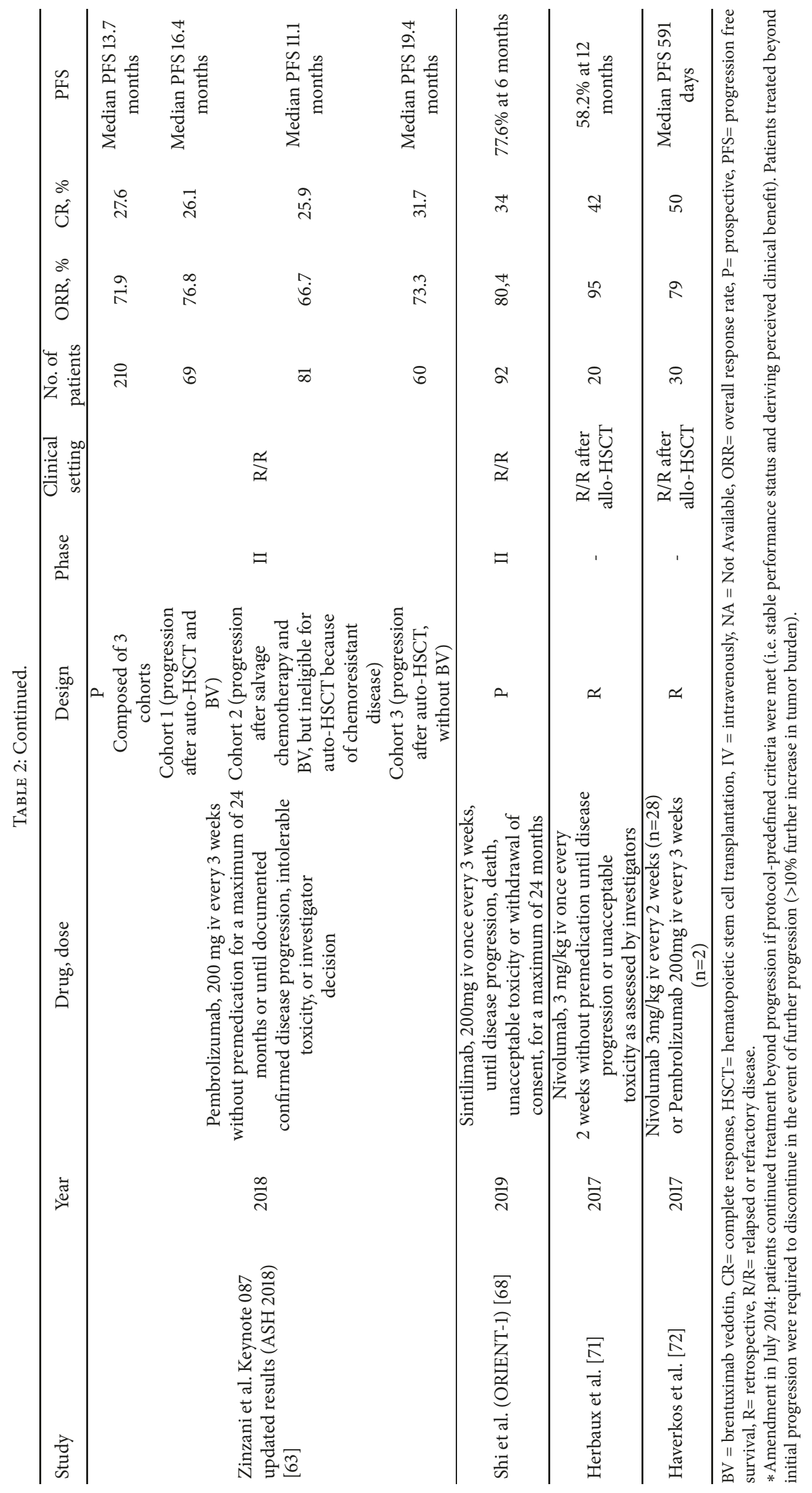


$71.9 \%$, with $27.6 \%$ of CR. Median PFS was 13.7 months. It seems also that cohort 2 (those with chemoresistant disease) had smaller ORR (66.7\% [95\%CI 55.3-76.8] vs. 76.8\% [95\%CI 65.1-86.1] in cohort 1 and 73.3\% [95\%CI 60.3-83.9] in cohort 3 ) and shorter PFS than the two other cohorts (11.1 months [95\%CI 7.6-13.8] vs. 16.4 [95\%CI 11.3-27.6] and 19.4 months [95\%CI 10.8-22.1], resp.) [63].

Based on these studies, nivolumab and pembrolizumab received FDA $[6,64,65]$ and $\mathrm{UE}[66,67]$ approvals for the treatment of patients with $\mathrm{cHL}$ who relapsed or progressed after auto-HSCT and posttransplantation BV.

More recently, another anti-PD-1 mAb, sintilimab, was tested in a phase 2 trial (ORIENT-1) [68]. Ninety-two patients with $\mathrm{R} / \mathrm{R}$ cHL were treated with sintilimab (200mg intravenous once every 3 weeks) until progression, death, unacceptable toxicity, or withdrawal of consent. ORR was $80.4 \%$, with $34 \%$ CR and a PFS of $77.6 \%$ at 6 months.

Of note, exclusion criteria in all the abovementioned studies comprised allo-HSCT. Indeed, two murine models raised the concern of increased GVHD-related mortality due to ICI exposure after allo-HSCT $[69,70]$.

Recently, a retrospective study on 20 patients treated with nivolumab after allo-HSCT was published [71]. Six patients experienced acute GVHD, and 2 patients deceased. Noteworthily, all of these patients had already suffered a previous episode of acute GVHD. In this cohort, nivolumab did not induce chronic GVHD and no flare phenomena was noted in four patients with previously documented chronic GVHD. In this study, nine patients showed a CR under nivolumab and 10 had a PR, with a PFS at 12 months of $58.2 \%$. Another retrospective study on 31 lymphoma patients (30 of them having $\mathrm{cHL})$ treated with nivolumab $(n=28)$ or pembrolizumab $(n=2)$ for relapse after allo-HSCT found an ORR of $77 \%$ (15 CR, 8 PR), but with 8 (26\%) GVHD-related deaths after anti-PD-1 therapy [72]. After initiation of antiPD-1, 17 patients developed GVHD (6 acute, 4 overlap, and 7 chronic). Median PFS was 591 days.

The impact of anti-PD-1 treatment on the risk of subsequent GVHD when administered before allo-HSCT is also a matter of debate. In a retrospective study published in 2017, Merryman et al. described a cohort of 39 patients with lymphoma (31 patients with $\mathrm{cHL}$ ), who received pembrolizumab or nivolumab and subsequently underwent alloHSCT (median time of 62 days, range 7-260, between end of immunotherapy and allo-HSCT) [73]. They found a 1year cumulative incidence of grade 3-4 acute GVHD of $23 \%$ and reported a PFS of $89 \%$. An analysis of circulating lymphocyte subsets in 17 patients showed, in comparison to controls, decreased numbers of PD-1+ T cells and lower ratios of T-regulatory cells to conventional CD4+ and CD8+cells, suggesting a possible long-term implication of prior ICI treatment on the immune system after allo-HSCT. A recent review summarized the published data on the impact of prior or subsequent anti-PD-1 therapy on GVHD in patients with lymphoma (the majority of whom were cHL) treated with an allo-HSCT [74]. Among the 107 patients who received ICI before allo-HSCT, acute and chronic GVHD surged in 56\% and $29 \%$ of patients, respectively. Mortality risk from GVHD was $11 \%$ in this study. One hundred and seventy-six patients treated with ICI after allo-HSCT were reported. The rates of acute and chronic GVHD were $14 \%$ and 5\%, respectively, with a mortality risk from GVHD of $7 \%$. In the absence of prospective data, recommendations for management of ICI before or after allo-HSCT are currently based on expert opinions [71]. These recommendations advocate empirically a 6-week interval between completion of anti-PD-1 therapy and allo-HSCT. In addition, these recommendations advise the use of reduced intensity conditioning regimen before alloHSCT in this heavily pretreated patient population.

3.2. Safety Profile. Adverse events consecutive to ICI are distinctly different from the ones related to conventional chemotherapy. The blockade of the immunological checkpoints with $\mathrm{mAb}$ can trigger autoimmune complications that can affect any organ. These so-called immune-related adverse events (irAEs) vary in incidence and spectrum of affected organ systems depending on the agents used. The incidence of irAES of any grade is variable and can affect up to half of patients under anti-PD-1 therapy, such as those in the ORIENT-1 trial [68]. The incidence of high-grade irAEs among the 651 patients with $\mathrm{R} / \mathrm{R} \mathrm{cHL}$ included in different clinical trials is summarized in Table 3. The most frequent grade 3-4 adverse events according to common terminology (CTCAE v.4.0) were gastrointestinal under the form of enterocolitis $(13 \%, 2 \%$, and $5 \%$ in patients treated with nivolumab, pembrolizumab, and sintilimab, resp.), pulmonary (2.1\% in total; including pneumonitis, dyspnea, and respiratory infections), and hepatic. Other reported highgrade adverse events encompassed general symptoms, such as fever and fatigue (1.2\%), but also mucocutaneous (1\%), cardiovascular $(0.4 \%)$, endocrine $(0.3 \%)$, rheumatological $(0.3 \%)$, and renal and electrolyte $(0.3 \%)$ disorders. These trials did not report fatal cases of irAEs and toxicities leading to treatment discontinuation were rare.

Hematologists should be aware of such possible complications in order to initiate early and adapted immunosuppressive treatments [75]. Most irAEs are reversible, except in the case of endocrine dysfunction, and are treated effectively by delaying the administration of the ICI and proper immunosuppressive treatments including corticosteroid and/or biological agents targeting key inflammatory cytokines such as interleukin-6 and tumor necrosis factor $\alpha$ [76].

3.3. Patients Not Responding to Anti-PD-1 Therapy. The radiological interpretation of tumor response is challenging in the context of immunotherapy. The assessment of tumor response using fluorodeoxyglucose-positron-emission tomography (FDG-PET) may lead, in some cases, to premature discontinuation of anti-PD-1 therapy, due to misleading imaging findings suggestive of disease progression. The immune activation and abundance of T-cell infiltration related to checkpoint inhibition has been linked to a phenomenon called "tumor flare" or "pseudoprogression" under the form of new lesions, or lesions increasing in size and metabolic activity [77]. A new biopsy or repeated imaging is advocated in these cases, mostly if the patient is experiencing clinical benefit from immunotherapy, before deciding to 


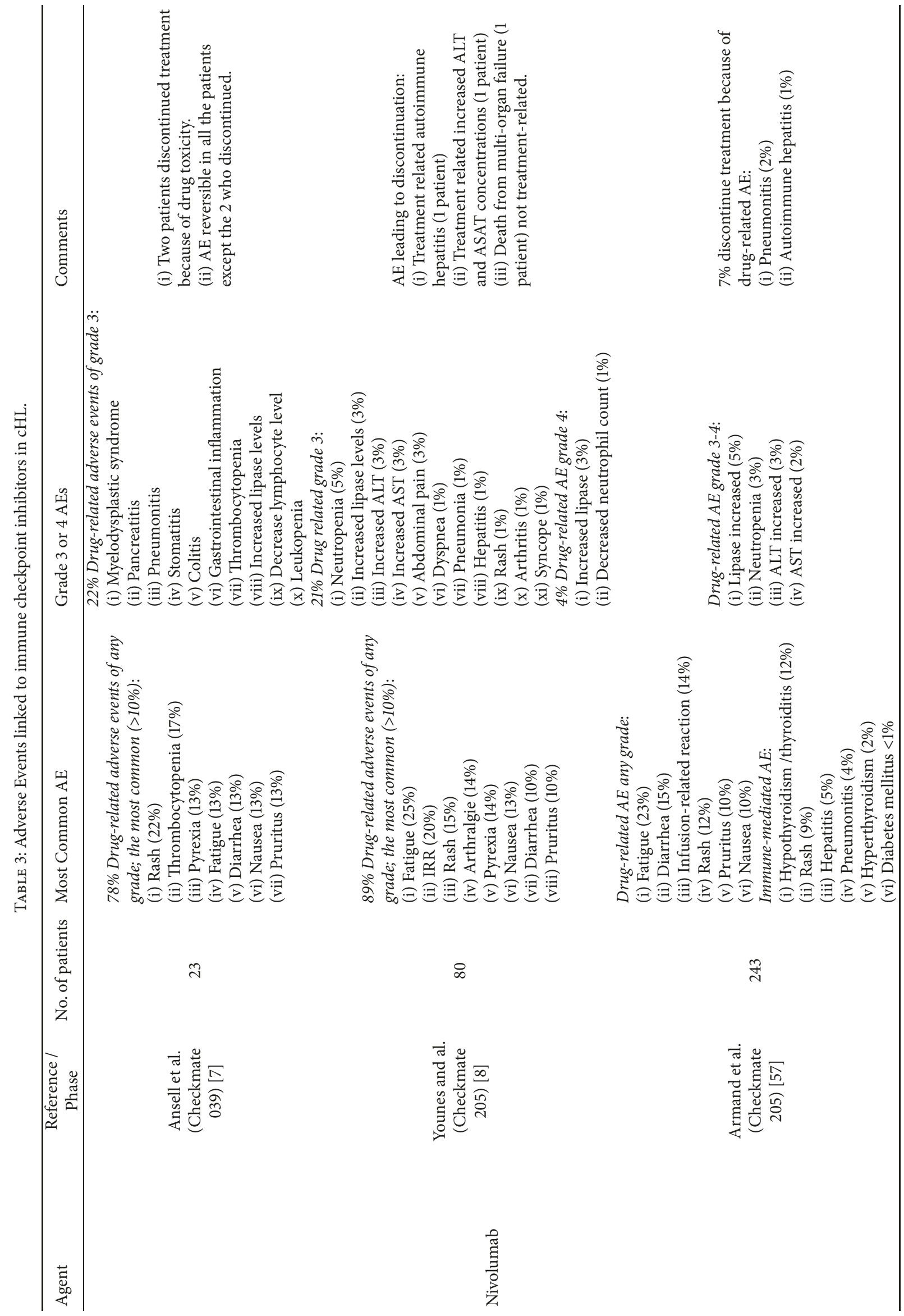




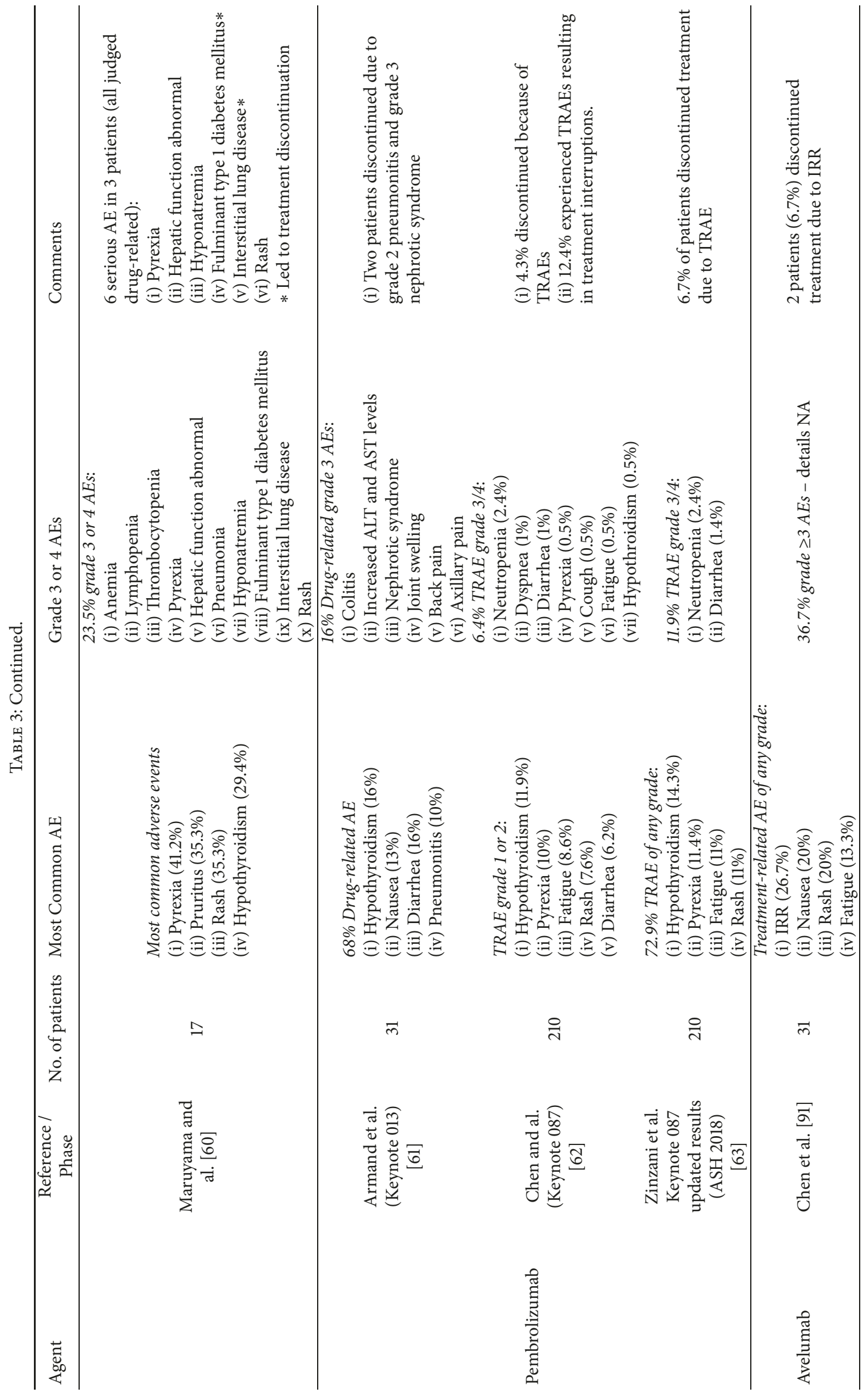




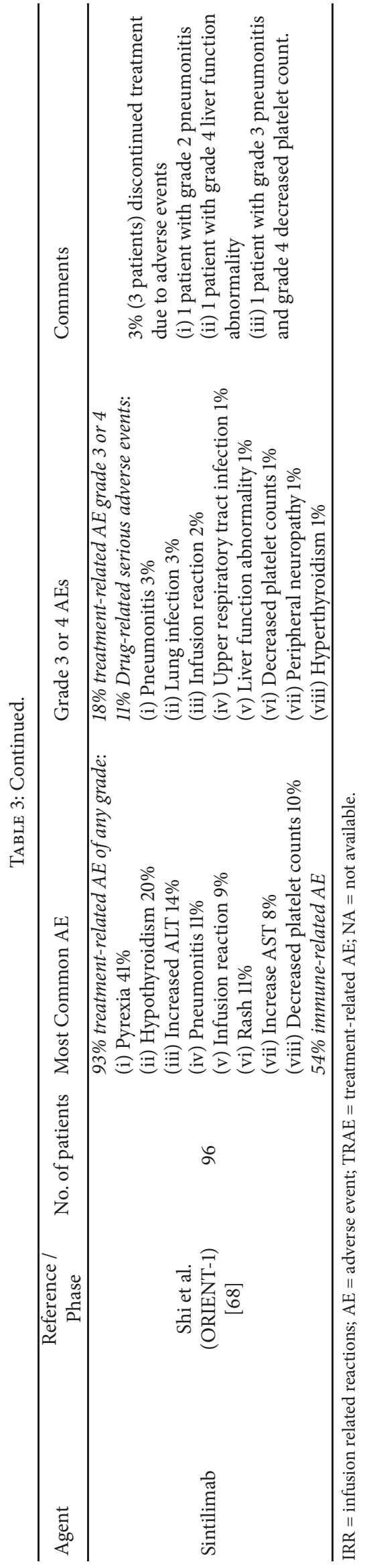


stop the treatment. Some patients showing these pseudoprogressions may experience late responses and even longlasting clinical benefit from anti-PD-1 therapy. The Lugano Classification lymphoma response criteria have been refined in 2016 to address this specific issue [78]. They determined the imaging criteria suggestive of pseudoprogression (in the absence of clinical deterioration) and classified these scenarios as "indeterminate responses" necessitating additional tests in order to identify a true progressive disease. If such an eventuality is confirmed, the treatment of patients who do not respond to anti-PD-1 therapy is a main concern. Rossi and colleagues retrospectively described the treatment of 30 patients with cHL highly pretreated and who failed anti-PD-1 therapy. Seventeen patients were treated with chemotherapy alone (group 1) and 7 with chemotherapy and anti-PD1 (group 2). ORR was $59 \%$ and $86 \%$, respectively. This observation suggests that anti-PD-1 therapy could resensitize tumor cells to chemotherapy-induced death [79].

\section{New Perspectives}

Future aim for patients with Hodgkin's disease, as well as with other lymphoma types and more broadly with cancers, is to provide them with more efficient treatment where side effects remain as minor as possible and manageable. In this endeavor, ICI has opened a new way to treat patients, at the price of awakening autoimmunity.

It can be foreseen that efficacy of actual checkpoint inhibition, targeting PD-1/PD-L1 (and CTLA-4), could be ameliorated when we contemplate and consider using some of the myriad of checkpoint molecules involved in the interactions of immune cells capable of killing lymphoma cells [80]. Such analysis has inherent complexity that resides not only within the number of checkpoints implicated, but also in their cell specificity and time-dependent expression.

One could foresee that combining ICI with classical chemotherapy, especially in heavily treated patients, would reduce the number of antigen-presenting and immune effector cells implicated in antilymphoma immune response awakened by ICI, and globally weakening ICI. Actually, ICI are prescribed mostly in heavily treated patients, but implementing ICI earlier in the course of the disease could be more beneficial in terms of treatment efficacy.

The Specificity of ICI efficiency resides in large part within the mutational burden of the tumor leading to neoantigen formation and presentation to immune cells. The antitumor response could be boosted with tumor-derived vaccines where patients are simultaneously receiving ICI. This alternative has been evaluated in preclinical models with success and is evaluated nowadays in some cancers, including lymphomas.

Finally, combining ICI with active and passive immunization could be most beneficial. This can be done by immunizing patients with tumoral neoantigen, or by transferring to patients tumoral neoantigen-specific cells (modified or not) followed by ICI to boost the antilymphoma response. Such an avenue is currently evaluated using $\mathrm{T}$ cells specific for $\mathrm{EBV}$ in combination with PD-1-PD-L1 blockade in $\mathrm{EBV}^{+}$lymphomas, including EBV ${ }^{+}$Hodgkin's disease (NCT02973113).
Immune checkpoint inhibitors are all implicated in classical as well as antilymphoma immune responses. A global analysis of ICI distribution, both spatially and with time, could help to design ICI administration with better specificity (i.e., targeting IC preferentially expressed in lymphoma versus healthy tissues) and efficacy (time and length of treatment). Such an analysis has already been undertaken without always-clear conclusions. This is probably due to the complexity of the antilymphoma immune response parameters. This complexity is best illustrated by the observations that ICI are even dependent on gut microbiota $[81,82]$. In the future, using a more complete picture of biomarkers would help.

Additionally, one could envisage using chemotherapeutic drugs with known immunomodulatory activities to enhance the efficacy of immune checkpoint inhibition, such as cyclophosphamide and anthracyclines. These chemotherapies can elicit an immunogenic cellular death (ICD) characterized by apoptotic bodies exposing calreticulin and releasing adenosine triphosphate (ATP) and high-mobility group box 1 protein (HMGB1) which act as an "eat me" signal towards adaptive immune cells [83-85]. Interestingly, hypomethylating agents have also been proposed to prime ICI. Interesting immunomodulatory properties of Bruton kinase inhibitors (BTK) such as ibrutinib have been pointed out in lymphoma and myeloma xenograft models [86]. Through the off-target inhibition of ITK (interleukin-2inducible T-cell kinase), ibrutinib is able to polarize T-cell response towards à Th1 tumor rejection-prone phenotype. This property is also amenable to ICI treatment combinations and is now being tested in R/R cHL (NCT02940301).

Combining radiotherapy with ICI has the potential to boost specifically the antilymphoma efficiency of ICI while preserving the development of an immune response. Indeed, radiotherapy leads to lymphoma cell death, liberates immune-activating chemokines and cytokines, activates dendritic cell and antigen presentation, and induces effector cells (such as CTL and NK cells) activation and proliferation [87]. ICI would boost the efficiency of these effector cells, resulting in preferentially local antilymphoma efficacy. In the context of CHL known to be most sensitive to radiotherapy, this combination may be highly synergistic and may conduct therapeutic strategies using lower doses of irradiation than those in current protocols.

Last, but not least, the education of medical specialists will have to be adapted according to these novel combination therapies in terms of both available therapies and follow-up exams. One example of the latter is that response is classically evaluated using radiological exams such as scanner in combination with FDG-PET. Such an exam cannot differentiate between residual lymphoma cells and activated and proliferating immune cells around and within the tumor. This can lead to a false positive evaluation of patient under ICI treatment.

\section{Conclusion}

Since the advent of modern oncology in the mid-fifties, cHL was the standard-bearer of chemotherapy and radiotherapy early successes. Thanks to these achievements, cHL is 
nowadays considered a curable disease. However, a subset of patients still suffer from relapses, and despite being considered a disease of young adults, senior populations also display a peak of incidence. These relapsing/refractory patients pose a challenge for oncohematologists, regarding the ways to achieve high response rates and cures in the young patient population, but also to limit toxicities while assuring the best quality of care in the older one. PD-1 inhibition is revealed to be a valuable therapeutic option in these situations, with a high response rate and a reasonable toxicity profile. However, practitioners should not underestimate autoimmune toxicities and should be aware of the need to initiate immediate interventions if they occur. With immunotherapy being a new treatment modality, clinicians should be trained to recognize these adverse events. Many questions are still open, such as if ICI will indeed be able to decrease long-term toxicities of current standard treatment protocols, replace radiotherapy, or continue as salvage therapy, but also regarding the interpretation of treatment responses. In this new era of immunotherapy, Hodgkin's disease is once again colliding with the history of oncology, as a paradigm of ICI sensitivity and a model for therapy development.

\section{Conflicts of Interest}

Authors declare that they have no conflicts of interest.

\section{Authors' Contributions}

Diego De Goycoechea, Gregoire Stalder, and Filipe Martins contributed equally to the review.

\section{Acknowledgments}

Filipe Martins thanks the Personalized Health and Related Technologies (PHRT) strategic focus area of the Swiss Federal Institutes of Technology (ETH) Domain and the Swiss Personalized Health Network (SPHN) initiative of the Swiss Academy of Medical Sciences for its support (project no. \#2017-407). The authors thank Mr. Roger Guindon for his help in the elaboration of Figure 1.

\section{References}

[1] R. L. Siegel, K. D. Miller, and A. Jemal, "Cancer statistics, 2017," CA: A Cancer Journal for Clinicians, vol. 67, no. 1, pp. 7-30, 2017.

[2] D. Reed, "On the pathological changes in Hodgkin's disease, with special reference to its relation to tuberculosis," The Johns Hopkins Hospital Reports, vol. 10, pp. 133-196, 1902.

[3] J. Treleaven, A. Gennery, J. Marsh et al., "Guidelines on the use of irradiated blood components prepared by the British Committee for Standards in Haematology blood transfusion task force," British Journal of Haematology, vol. 152, no. 1, pp. 35-51, 2011.

[4] I. Árpád, S. Zsófia, U. Miklós, M. Ferenc, J. Ádám, and M. Zsófia, "Treatment of relapsed Hodgkin lymphoma after autologous stem cell Transplantation,” Orvosi Hetilap, vol. 158, no. 34, pp. 1338-1345, 2017.
[5] A. Rashidi, M. Ebadi, and A. F. Cashen, "Allogeneic hematopoietic stem cell transplantation in Hodgkin lymphoma: A systematic review and meta-analysis," Bone Marrow Transplantation, vol. 51, no. 4, pp. 521-528, 2016.

[6] Y. L. Kasamon, R. A. De Claro, Y. Wang, Y. L. Shen, A. T. Farrell, and R. Pazdur, "FDA approval summary: Nivolumab for the treatment of relapsed or progressive classical hodgkin lymphoma," The Oncologist, vol. 22, no. 5, pp. 585-591, 2017.

[7] S. M. Ansell, A. M. Lesokhin, I. Borrello et al., "PD-1 blockade with nivolumab in relapsed or refractory Hodgkin's lymphoma," The New England Journal of Medicine, vol. 372, no. 4, pp. 311-319, 2015.

[8] A. Younes, A. Santoro, M. Shipp et al., "Nivolumab for classical Hodgkin's lymphoma after failure of both autologous stemcell transplantation and brentuximab vedotin: a multicentre, multicohort, single-arm phase 2 trial," The Lancet Oncology, vol. 17, no. 9, pp. 1283-1294, 2016.

[9] R. Ramchandren, M. A. Fanale, A. Rueda et al., "Nivolumab for newly diagnosed advanced-stage classical hodgkin lymphoma (cHL): results from the phase 2 checkmate 205 study," Blood, vol. 130, p. 651, 2017.

[10] H. Savas, P. Allen, A. Evens et al., "A phase II study of sequential pembrolizumab (pem) followed by avd for frontline treatment of classical hodgkin lymphoma (CHL): quantifying response following PEM monotherapy with FDG-PET-derived metabolic tumor volume and total lesion glycolysis," Blood, vol. 132, p. 1651, 2018.

[11] KM. Fox, N. C. Josephson, and A. Richhariya, "Toxicity burden of bleomycin treatment in hodgkin lymphoma: a systematic literature review," Blood, vol. 128, no. 22, p. 3566, 2016.

[12] P. L. Zinzani, V. Ribrag, C. H. Moskowitz et al., "Safety and tolerability of pembrolizumab in patients with relapsed/refractory primary mediastinal large B-cell lymphoma," Blood, vol. 130, no. 3, pp. 267-270, 2017.

[13] C. Melani, A. Major, J. Schowinsky et al., "PD-1 blockade in mediastinal gray-zone lymphoma," The New England Journal of Medicine, vol. 377, no. 1, pp. 89-91, 2017.

[14] L. Nayak, F. M. Iwamoto, A. Lacasce et al., "PD-1 blockade with nivolumab in relapsed/refractory primary central nervous system and testicular lymphoma," Blood, vol. 129, no. 23, pp. 3071-3073, 2017.

[15] R. W. Merryman, P. Armand, K. T. Wright, and S. J. Rodig, "Checkpoint blockade in Hodgkin and non-Hodgkin lymphoma," Blood Advances, vol. 1, no. 26, pp. 2643-2654, 2017.

[16] R. Küppers and K. Rajewsky, "The origin of Hodgkin and Reed/Sternberg cells in Hodgkin's disease," Annual Review of Immunology, vol. 16, pp. 471-493, 1998.

[17] M.-L. Hansmann and K. Willenbrock, "The WHO classification of Hodgkin's lymphoma and its molecular pathological relevance," Der Pathologe, vol. 23, no. 3, pp. 207-218, 2002.

[18] D. Hasenclever and V. Diehl, "A prognostic score for advanced Hodgkin's disease," The New England Journal of Medicine, vol. 339, no. 21, pp. 1506-1514, 1998.

[19] P. J. Bröckelmann, M. K. Angelopoulou, and T. P. Vassilakopoulos, "Prognostic factors in Hodgkin lymphoma," Seminars in Hematology, vol. 53, no. 3, pp. 155-164, 2016.

[20] F. Wein and R. Küppers, "The role of $t$ cells in the microenvironment of hodgkin lymphoma," Journal of Leukocyte Biology, vol. 99, no. 1, pp. 45-50, 2016.

[21] C. Steidl, J. M. Connors, and R. D. Gascoyne, "Molecular pathogenesis of hodgkin's lymphoma: Increasing evidence of 
the importance of the microenvironment," Journal of Clinical Oncology, vol. 29, no. 14, pp. 1812-1826, 2011.

[22] M. Fischer, M. Juremalm, N. Olsson et al., "Expression of CCL5/RANTES by Hodgkin and reed-sternberg cells and its possible role in the recruitment of mast cells into lymphomatous tissue," International Journal of Cancer, vol. 107, no. 2, pp. 197201, 2003.

[23] M. Niens, L. Visser, I. M. Nolte et al., "Serum chemokine levels in Hodgkin lymphoma patients: Highly increased levels of CCL17 and CCL22," British Journal of Haematology, vol. 140, no. 5, pp. 527-536, 2008.

[24] A. Van Den Berg, L. Visser, and S. Poppema, "High expression of the CC chemokine TARC in Reed-Sternberg cells: a possible explanation for the characteristic T-cell infiltrate in Hodgkin's lymphoma," The American Journal of Pathology, vol. 154, no. 6, pp. 1685-1691, 1999.

[25] D. Nagel, M. Vincendeau, A. C. Eitelhuber, and D. Krappmann, "Mechanisms and consequences of constitutive NF- $\kappa \mathrm{B}$ activation in B-cell lymphoid malignancies," Oncogene, vol. 33, no. 50, pp. 5655-5665, 2014.

[26] A. Chiu, W. Xu, B. He et al., "Hodgkin lymphoma cells express TACI and BCMA receptors and generate survival and proliferation signals in response to BAFF and APRIL," Blood, vol. 109, no. 2, pp. 729-739, 2007.

[27] D. Aldinucci, A. Gloghini, A. Pinto, A. Colombatti, and A. Carbone, "The role of CD40/CD40L and interferon regulatory factor 4 in Hodgkin lymphoma microenvironment," Leukemia \& Lymphoma, vol. 53, no. 2, pp. 195-201, 2012.

[28] P. K. Panjwani, V. Charu, M. DeLisser, H. Molina-Kirsch, Y. Natkunam, and S. Zhao, "Programmed death-1 ligands PDL1 and PD-L2 show distinctive and restricted patterns of expression in lymphoma subtypes," Human Pathology, vol. 71, pp. 91-99, 2018.

[29] M. R. Green, S. Monti, S. J. Rodig et al., "Integrative analysis reveals selective 9p24.1 amplification, increased PD-1 ligand expression, and further induction via JAK2 in nodular sclerosing Hodgkin lymphoma and primary mediastinal large B-cell lymphoma," Blood, vol. 116, no. 17, pp. 3268-3277, 2010.

[30] C. Mancao and W. Hammerschmidt, "Epstein-Barr virus latent membrane protein $2 \mathrm{~A}$ is a $\mathrm{B}$-cell receptor mimic and essential for B-cell survival," Blood, vol. 110, no. 10, pp. 3715-3721, 2007.

[31] G. Massini, D. Siemer, and S. Hohaus, "EBV in Hodgkin Lymphoma," Mediterranean Journal of Hematology and Infectious Diseases, vol. 1, no. 2, Article ID e2009013, 2009.

[32] M. R. Green, S. Rodig, P. Juszczynski et al., "Constitutive AP-1 activity and EBV infection induce PD-11 in Hodgkin lymphomas and posttransplant lymphoproliferative disorders: Implications for targeted therapy," Clinical Cancer Research, vol. 18, no. 6, pp. 1611-1618, 2012.

[33] C. Penafuerte, L. A. Perez-Quintero, V. Vinette, T. Hatzihristidis, and M. L. Tremblay, "Mining the complex family of protein tyrosine phosphatases for checkpoint regulators in immunity," Current Topics in Microbiology and Immunology, vol. 410, pp. 191-214, 2017.

[34] C. R. Calvo, D. Amsen, and A. M. Kruisbeek, "Cytotoxic T lymphocyte antigen 4 (CTLA-4) interferes with extracellular signal-regulated kinase (ERK) and jun NH2-terminal kinase (JNK) activation, but does not affect phosphorylation of T cell receptor $\zeta$ and ZAP70," The Journal of Experimental Medicine, vol. 186, no. 10, pp. 1645-1653, 1997.

[35] B. M. Carreno, F. Bennett, T. A. Chau et al., "CTLA-4 (CD152) Can Inhibit T Cell Activation by Two Different Mechanisms
Depending on Its Level of Cell Surface Expression," The Journal of Immunology, vol. 165, no. 3, pp. 1352-1356, 2000.

[36] T. Takahashi, T. Tagami, S. Yamazaki et al., "Immunologic self-tolerance maintained by $\mathrm{CD} 25^{+} \mathrm{CD} 4^{+}$regulatory $\mathrm{T}$ cells constitutively expressing cytotoxic $\mathrm{T}$ lymphocyte-associated antigen 4," The Journal of Experimental Medicine, vol. 192, no. 2, pp. 303-310, 2000.

[37] J. Larkin, D. Minor, S. D’Angelo et al., "Overall survival in patients with advanced melanoma who received nivolumab versus investigator's choice chemotherapy in CheckMate 037: A Randomized, Controlled, Open-Label Phase III Trial," Journal of Clinical Oncology, vol. 36, no. 4, pp. 383-390, 2018.

[38] R. J. Motzer, N. M. Tannir, D. F. McDermott et al., "Nivolumab plus Ipilimumab versus sunitinib in advanced renal-cell carcinoma," New England Journal of Medicine, vol. 378, no. 14, pp. 1277-1290, 2018.

[39] M. D. Hellmann, T.-E. Ciuleanu, A. Pluzanski et al., "Nivolumab plus ipilimumab in lung cancer with a high tumor mutational burden," The New England Journal of Medicine, vol. 378, no. 22, pp. 2093-2104, 2018.

[40] L. el Halabi, J. Adam, V. Marty et al., "Strong expression of the immune checkpoint regulators LAG3 and Tim3 in hodgkin lymphoma," Blood, vol. 128, p. 2952, 2016.

[41] C.-T. Huang, C. J. Workman, D. Flies et al., "Role of LAG-3 in regulatory T cells," Immunity, vol. 21, no. 4, pp. 503-513, 2004.

[42] J. F. Grosso, C. C. Kelleher, T. J. Harris et al., "LAG-3 regulates $\mathrm{CD}^{+} \mathrm{T}$ cell accumulation and effector function in murine self- and tumor-tolerance systems," The Journal of Clinical Investigation, vol. 117, no. 11, pp. 3383-3392, 2007.

[43] C. J. Workman, K. J. Dugger, and D. A. A. Vignali, "Cutting edge: Molecular analysis of the negative regulatory function of lymphocyte activation gene-3," The Journal of Immunology, vol. 169, no. 10, pp. 5392-5395, 2002.

[44] C. Y. Ok and K. H. Young, "Checkpoint inhibitors in hematological malignancies," Journal of Hematology \& Oncology, vol. 10, no. 1, p. 103, 2017.

[45] L. Monney, C. A. Sabatos, J. L. Gaglia et al., "Thl-specific cell surface protein Tim-3 regulates macrophage activation and severity of an autoimmune disease," Nature, vol. 415, no. 6871, pp. 536-541, 2002.

[46] M. Rangachari, C. Zhu, K. Sakuishi et al., "Bat3 promotes T cell responses and autoimmunity by repressing Tim-3-mediated cell death and exhaustion," Nature Medicine, vol. 18, no. 9, pp. 13941400, 2012.

[47] M. G. Roemer, R. H. Advani, R. A. Redd et al., "Classical Hodgkin Lymphoma with Reduced 2M/MHC Class I Expression Is Associated with Inferior Outcome Independent of 9p24.1 Status," Cancer Immunology Research, vol. 4, no. 11, pp. 910-916, 2016.

[48] C. Steidl, S. P. Shah, B. W. Woolcock et al., "MHC class II transactivator CIITA is a recurrent gene fusion partner in lymphoid cancers," Nature, vol. 471, no. 7338, pp. 377-383, 2011.

[49] M. Nijland, R. N. Veenstra, L. Visser et al., "HLA dependent immune escape mechanisms in B-cell lymphomas: Implications for immune checkpoint inhibitor therapy?" OncoImmunology, vol. 6, no. 4, Article ID e1295202, 2017.

[50] F. Vari, D. Arpon, C. Keane et al., "Immune evasion via $\mathrm{PD}-1 / \mathrm{PD}-\mathrm{Ll}$ on NK cells and monocyte/macrophages is more prominent in Hodgkin lymphoma than DLBCL," Blood, vol. 131, no. 16, pp. 1809-1819, 2018. 
[51] E. M. Maggio, A. Van den Berg, D. De Jong, A. Diepstra, and S. Poppema, "Low frequency of FAS mutations in ReedSternberg cells of Hodgkin's lymphoma," The American Journal of Pathology, vol. 162, no. 1, pp. 29-35, 2003.

[52] M. R. Zocchi, S. Catellani, P. Canevali et al., "High ERp5/ ADAM10 expression in lymph node microenvironment and impaired NKG2D ligands recognition in Hodgkin lymphomas," Blood, vol. 119, no. 6, pp. 1479-1489, 2012.

[53] N. Rouas-Freiss, P. Moreau, J. LeMaoult, and E. D. Carosella, "The dual role of HLA-G in cancer," Journal of Immunology Research, vol. 2014, Article ID 359748, 10 pages, 2014.

[54] B. K. Kaiser, D. Yim, I.-T. Chow et al., "Disulphide-isomeraseenabled shedding of tumour-associated NKG2D ligands," Nature, vol. 447, no. 7143, pp. 482-486, 2007.

[55] E. B. Wilson, J. J. El-Jawhari, A. L. Neilson et al., "Human tumour immune evasion via TGF- $\beta$ blocks NK cell activation but not survival allowing therapeutic restoration of anti-tumour activity," PLoS ONE, vol. 6, no. 9, Article ID e22842, 2011.

[56] M. Gotti, M. Nicola, M. Lucioni et al., "Independent prognostic impact of tumour-infiltrating macrophages in early-stage Hodgkin's lymphoma," Hematological Oncology, vol. 35, no. 3, pp. 296-302, 2017.

[57] P. Armand, A. Engert, A. Younes et al., "Nivolumab for relapsed/refractory classic hodgkin lymphoma after failure of autologous hematopoietic cell transplantation: Extended follow-up of the multicohort single-arm phase II checkmate 205 trial," Journal of Clinical Oncology, vol. 36, no. 14, pp. 1428-1439, 2018.

[58] P. Armand, A. Engert, A. Younes et al., "Nivolumab for relapsed or refractory classical hodgkin lymphoma (cHL) after autologous hematopoietic cell transplantation (auto-HCT): extended follow-up of the phase 2 single-arm checkmate 205 study," Blood, vol. 132, p. 2897, 2018.

[59] J. B. Cohen, J. Kuruvilla, A. Engert et al., "Nivolumab treatment beyond investigator-assessed progression: extended follow-up in patients with relapsed/refractory classical hodgkin lymphoma from the phase 2 checkmate 205 study," Blood, vol. 132, p. 2932, 2018.

[60] D. Maruyama, K. Hatake, T. Kinoshita et al., "Multicenter phase II study of nivolumab in Japanese patients with relapsed or refractory classical Hodgkin lymphoma," Cancer Science, vol. 108, no. 5, pp. 1007-1012, 2017.

[61] P. Armand, M. A. Shipp, V. Ribrag et al., "Programmed death1 blockade with pembrolizumab in patients with classical hodgkin lymphoma after brentuximab vedotin failure," Journal of Clinical Oncology, vol. 34, no. 31, pp. 3733-3739, 2016.

[62] R. Chen, P. L. Zinzani, M. A. Fanale et al., "Phase II study of the efficacy and safety of pembrolizumab for relapsed/refractory classic Hodgkin Lymphoma," Journal of Clinical Oncology, vol. 35, no. 19, pp. 2125-2132, 2017.

[63] P. L. Zinzani, R. W. Chen, H. J. Lee, and P. Armand, “Two-year follow-up of keynote-087 study: pembrolizumab monotherapy in relapsed/refractory classic hodgkin lymphoma," Blood, vol. 132, p. 2900, 2018.

[64] US Food and Drug Administration, "Approved Drugs - Nivolumab (Opdivo) for Hodgkin Lymphoma," (accessed 14 February 2019), https://www.fda.gov/Drugs/InformationOnDrugs/ApprovedDrugs/ucm501412.htm.

[65] US Food and Drug Administration, "Approved Drugs - Pembrolizumab (KEYTRUDA) for Classical Hodgkin Lymphoma," (accessed 14 February 2019), https://www.fda.gov/Drugs/ InformationOnDrugs/ApprovedDrugs/ucm546893.htm.
[66] European Medicines Agency, "Keytruda," https://www.ema .europa.eu/en/medicines/human/EPAR/keytruda, (accessed 14 February 2019).

[67] Europena Medicines Agency, "Opdivo," https://www.ema .europa.eu/en/medicines/human/EPAR/opdivo, (accessed 14 February 2019).

[68] Y. Shi, H. Su, Y. Song et al., "Safety and activity of sintilimab in patients with relapsed or refractory classical Hodgkin lymphoma (ORIENT-1): a multicentre, single-arm, phase 2 trial," The Lancet Haematology, vol. 6, no. 1, pp. e12-e19, 2019.

[69] B. R. Blazar, B. M. Carreno, A. Panoskaltsis-Mortari et al., "Blockade of Programmed death-1 engagement accelerates graft-versus-host disease lethality by an ifn-gamma-dependent mechanism," The Journal of Immunology, vol. 171, no. 3, pp. 1272-1277, 2003.

[70] A. Saha, K. Aoyama, P. A. Taylor et al., "Host programmed death ligand 1 is dominant over programmed death ligand 2 expression in regulating graft-versus-host disease lethality," Blood, vol. 122, no. 17, pp. 3062-3073, 2013.

[71] C. Herbaux, J. Gauthier, P. Brice et al., "Efficacy and tolerability of nivolumab after allogeneic transplantation for relapsed Hodgkin lymphoma," Blood, vol. 129, no. 18, pp. 2471-2478, 2017.

[72] B. M. Haverkos, D. Abbott, M. Hamadani et al., "PD-1 blockade for relapsed lymphoma post-allogeneic hematopoietic cell transplant: high response rate but frequent GVHD," Blood, vol. 130, no. 2, pp. 221-228, 2017.

[73] R. W. Merryman, H. T. Kim, P. L. Zinzani et al., "Safety and efficacy of allogeneic hematopoietic stem cell transplant after PD-1 blockade in relapsed/refractory lymphoma," Blood, vol. 129, no. 10, pp. 1380-1388, 2017.

[74] A. Ijaz, A. Y. Khan, S. U. Malik et al., "Significant risk of graftversus-host disease with exposure to checkpoint inhibitors before and after allogeneic transplantation," Biology of Blood and Marrow Transplantation, vol. 25, no. 1, pp. 94-99, 2019.

[75] F. Martins, G. P. Sykiotis, M. Maillard et al., "New therapeutic perspectives to manage refractory immune checkpoint-related toxicities," The Lancet Oncology, vol. 20, no. 1, pp. e54-e64, 2019.

[76] M. A. Postow, R. Sidlow, and M. D. Hellmann, "Immune-related adverse events associated with immune checkpoint blockade," The New England Journal of Medicine, vol. 378, no. 2, pp. 158$168,2018$.

[77] L. Beer, M. Hochmair, and H. Prosch, "Pitfalls in the radiological response assessment of immunotherapy," Memo - Magazine of European Medical Oncology, vol. 11, no. 2, pp. 138-143, 2018.

[78] B. D. Cheson, S. Ansell, L. Schwartz et al., "Refinement of the Lugano Classification lymphoma response criteria in the era of immunomodulatory therapy," Blood, vol. 128, no. 21, pp. 24892496, 2016.

[79] C. Rossi, J. Gilhodes, M. Maerevoet et al., "Efficacy of chemotherapy or chemo-anti-PD-1 combination after failed anti-PD-1 therapy for relapsed and refractory hodgkin lymphoma: A series from lysa centers," American Journal of Hematology, vol. 93, no. 8, pp. 1042-1049, 2018.

[80] I. Hude, S. Sasse, A. Engert, and P. J. Bröckelmann, "The emerging role of immune checkpoint inhibition in malignant lymphoma," Haematologica, vol. 102, no. 1, pp. 30-42, 2017.

[81] M. Vétizou, J. M. Pitt, R. Daillère et al., "Anticancer immunotherapy by CTLA-4 blockade relies on the gut microbiota," Science, vol. 350, no. 6264, pp. 1079-1084, 2015.

[82] A. Sivan, L. Corrales, N. Hubert et al., "Commensal Bifidobacterium promotes antitumor immunity and facilitates anti-PDL1 efficacy," Science, vol. 350, no. 6264, pp. 1084-1089, 2015. 
[83] M. Obeid, A. Tesniere, F. Ghiringhelli et al., "Calreticulin exposure dictates the immunogenicity of cancer cell death," Nature Medicine, vol. 13, no. 1, pp. 54-61, 2007.

[84] I. Martins, Y. Wang, M. Michaud et al., "Molecular mechanisms of ATP secretion during immunogenic cell death," Cell Death \& Differentiation, vol. 21, no. 1, pp. 79-91, 2014.

[85] M. T. Lotze and K. J. Tracey, "High-mobility group box 1 protein (HMGB1): nuclear weapon in the immune arsenal," Nature Reviews Immunology, vol. 5, no. 4, pp. 331-342, 2005.

[86] I. Sagiv-Barfi, H. E. K. Kohrt, D. K. Czerwinski, P. P. Ng, B. Y. Chang, and R. Levy, "Therapeutic antitumor immunity by checkpoint blockade is enhanced by ibrutinib, an inhibitor of both BTK and ITK," Proceedings of the National Acadamy of Sciences of the United States of America, vol. 112, no. 9, pp. E966E972, 2015.

[87] A. B. Sharabi, M. Lim, T. L. DeWeese, and C. G. Drake, "Radiation and checkpoint blockade immunotherapy: Radiosensitisation and potential mechanisms of synergy," The Lancet Oncology, vol. 16, no. 13, pp. e498-e509, 2015.

[88] C. S. Verbeke, U. Wenthe, R. Grobholz, and H. Zentgraf, "Fas ligand expression in Hodgkin lymphoma," The American Journal of Surgical Pathology, vol. 25, no. 3, pp. 388-394, 2001.

[89] C. L. Batlevi, Y. Kasamon, R. G. Bociek et al., "ENGAGE501: Phase II study of entinostat (SNDX-275) in relapsed and refractory hodgkin lymphoma," Haematologica, vol. 101, no. 8, pp. 968-975, 2016.

[90] A. Younes, A. Sureda, D. Ben-Yehuda et al., "Panobinostat in patients with relapsed/refractory Hodgkin's lymphoma after autologous stem-cell transplantation: results of a phase II study," Journal of Clinical Oncology, vol. 30, no. 18, pp. 2197-2203, 2012.

[91] R. Chen, A. Gibb, G. Collins et al., "Blockade of the PD1 checkpoint with anti-PD-L1 antibody avelumab is sufficient for clinical activity in relapsed/refractory classical Hodgkin lymphoma (CHL)," Hematological Oncology, vol. 35, no. S2, p. 67, 2017. 


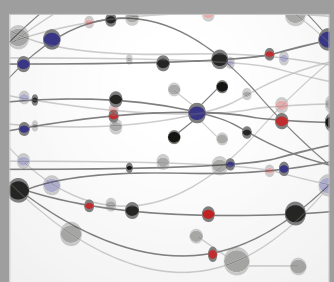

The Scientific World Journal
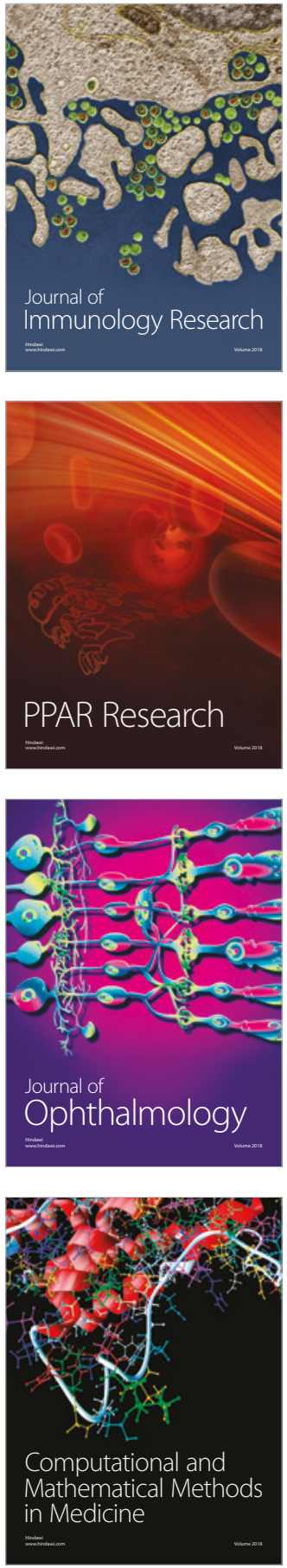

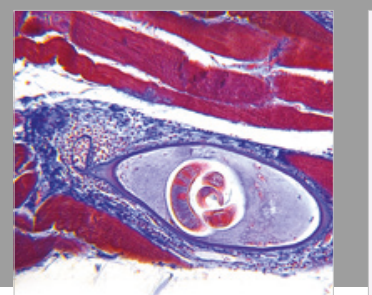

Gastroenterology Research and Practice

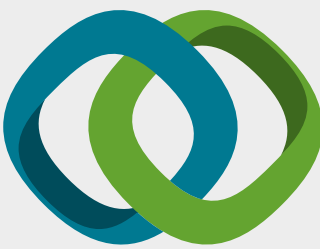

\section{Hindawi}

Submit your manuscripts at

www.hindawi.com
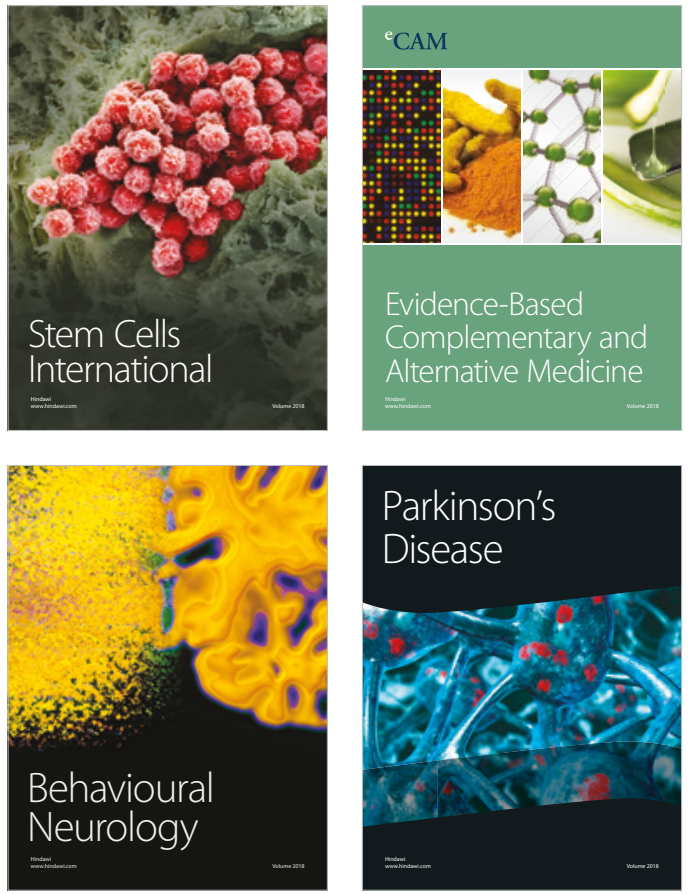

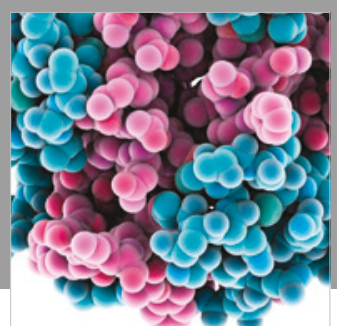

ournal of

Diabetes Research

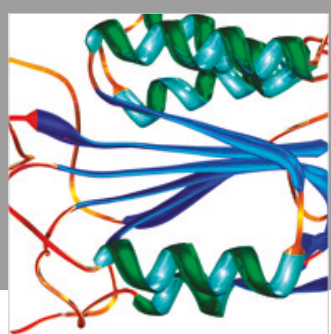

Disease Markers
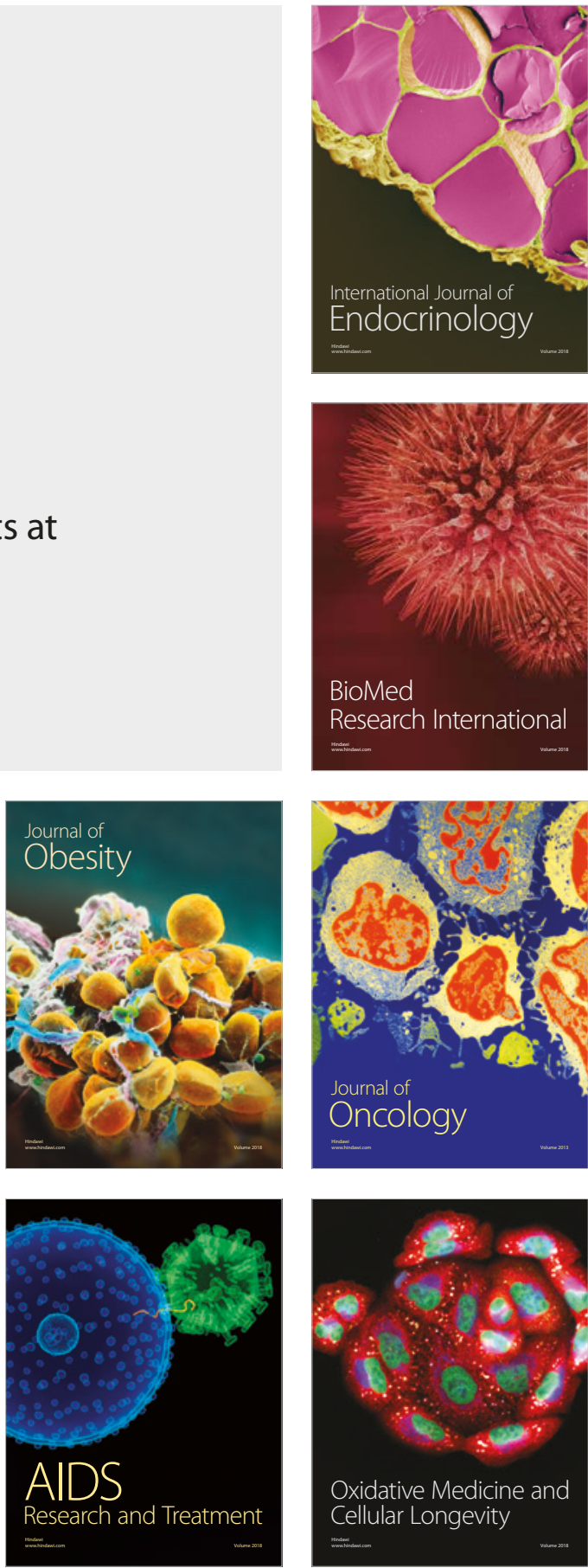